\title{
Efecto de la liberación controlada de nitrógeno sobre la fermentación y la degradabilidad in situ de Cynodon dactylon
}

\section{Effect of controlled-release of nitrogen on fermentation and in situ digestibility of Cynodon dactylon}

\author{
Álvaro Ojeda, ${ }^{1 *}$ Ph.D, Miguel Reyes, ${ }^{2}$ Ing, Williams Rodríguez, ${ }^{2}$ Ing.
}

${ }^{1}$ Universidad Central de Venezuela, Facultad de Agronomía, Instituto de Producción Animal, Maracay, Venezuela. ${ }^{2}$ Ejercicio particular. *Correspondencia: ajojeda99@yahoo.com

Recibido: Abril de 2011; Aceptado: Enero de 2012.

\begin{abstract}
RESUMEN
Objetivo. Evaluar el efecto de una fuente no proteica de liberación controlada de nitrógeno (NnpLC) sobre algunos parámetros de la fermentación ruminal y degradabilidad in situ de Cynodon dactylon. Materiales y métodos. 4 vacas fistuladas al rumen alimentadas con una dieta base de heno de Cynodon dactylon (4.8\% proteína cruda y $78.4 \%$ fibra detergente neutra), $1 \mathrm{~kg}$ de melaza de caña y $55 \mathrm{~g}$ de mezcla mineral (tratamiento Control), y tratamientos experimentales con adición a la dieta base de $150 \mathrm{~g}$ urea (Urea), sustitución de Urea por NnpLC a razón de 50\% del aporte de nitrógeno (Urea/ NnpLC) y $183 \mathrm{~g} \mathrm{NnpLC} \mathrm{(NnpLC).} \mathrm{En} \mathrm{un} \mathrm{Cuadrado} \mathrm{Latino} \mathrm{4x4} \mathrm{y} \mathrm{períodos} \mathrm{de} 17$ días, se registró consumo del día 7 al 14 . El día 15 fueron tomadas muestras seriadas de contenido ruminal para evaluar $\mathrm{pH}$, nitrógeno amoniacal $\left(\mathrm{N}-\mathrm{NH}_{3}\right)$ y ácidos grasos volátiles. La degradabilidad de la materia orgánica $\left(\mathrm{DMO}_{48}\right)$ y fibra detergente neutro $\left(\mathrm{DFND}_{48}\right)$ a las $48 \mathrm{~h}$ fueron medidas con bolsas de nylon. Resultados. No hubo diferencias $(p>0.05)$ en consumo de materia seca $(8.2 \pm 0.35 \mathrm{kgMS} / \mathrm{animal} /$ día), $\mathrm{pH}(6.1 \pm 0.21), \mathrm{DMO}_{48}(52.2 \pm 6.2 \%)$ y $\mathrm{DFND}_{48}(30.1 \pm 2.8 \%)$; aunque hubo diferencias $(\mathrm{p}<0.01)$ en valores medios de $\mathrm{N}-\mathrm{NH}_{3}(19.1,166.7,181.6$ y $281.8 \mathrm{mg} / \mathrm{L}$; respectivamente). NnpLC incrementó $(\mathrm{p}<0.05)$ el ácido propiónico $(27.3 \%)$, redujo el $\mathrm{T}_{1 / 2}(13.2 \%)$ y optimizó la relación P:E $(22.0 \pm$ $0.76)$. Conclusiones. El uso de una fuente NnpLC generó un perfil de ácidos grasos volátiles con patrón gluconeogénico, optimizó la concentración de $\mathrm{N}-\mathrm{NH}_{3}$ y mejoró la relación $\mathrm{P}$ : $\mathrm{E}$, por lo que debe considerarse una alternativa para manipular el medio ambiente ruminal de vacunos alimentados con recursos fibrosos.
\end{abstract}

Palabras clave: Ácidos grasos, nitrógeno amoniacal, rumen, urea (Fuente: AGROVOC). 


\section{ABSTRACT}

Objective. To evaluate the effect of a controlled-release non protein nitrogen source (CRNpn) on some parameters of rumen fermentation and in situ digestibility of Cynodon dactylon. Materials and methods. Four rumen fistulated cows fed a basal diet with Cynodon dactylon hay (4.8\% crude protein and $78.4 \%$ neutral detergent fiber), $1 \mathrm{~kg}$ sugar cane molasses and $55 \mathrm{~g}$ mineral mix (Control), and experimental treatment plus $150 \mathrm{~g}$ urea (Urea), replaced Urea with CRNpn by 50\% of the nitrogen contribution (Urea/CRNpn) and $183 \mathrm{~g}$ CRNpn (CRNpn), in a 4x4 Latin Square with periods of 17 days. Intake was measured between days 7 and 14, serial rumen content samples were taken to measure $\mathrm{pH}$, ammonia nitrogen $\left(\mathrm{N}-\mathrm{NH}_{3}\right)$ and volatile fatty acids on day 15 . Organic matter (OMD48) and neutral detergent fiber (NDF48) digestibility were measured with nylon bags at $48 \mathrm{~h}$. Results. No differences ( $p>0.05)$ on dry matter intake $(8.2 \pm 0.35 \mathrm{kgDM} /$ animal/day), ruminal $\mathrm{pH}(6.1 \pm 0.21), \mathrm{OMD} 48(52.2 \pm 6.2 \%)$ and NDF48 (30.1 $2.8 \%)$ were observed. However, there were differences $(\mathrm{p}<0.01)$ in $\mathrm{N}-\mathrm{NH}_{3}$ daily averages $(19.1,166.7,181.6$ and $281.8 \mathrm{mg} / \mathrm{L}$; respectively). CRNpn increased $(p<0.05)$ the propionic acid $(27.3 \%)$, reduced $T_{1 / 2}(13.2 \%)$ and optimized the P:E relation (22.0 \pm 0.76$)$. Conclusions. The use of a CRNpn source generated a volatile fatty acid profile with gluconeogenic pattern, optimized the $\mathrm{N}-\mathrm{NH}_{3}$ concentration and improved the P:E relation, and therefore may be considered as an alternative to manipulate the ruminal environment of cattle fed with fibrous resources.

Key words: Ammonia nitrogen, fatty acids, rumen, urea (Source: AGROVOC).

\section{INTRODUCCIÓN}

La proteína microbiana representa entre 70 y $90 \%$ de los aminoácidos que alcanzan el duodeno en un vacuno en pastoreo, y su síntesis requiere de aportes ruminales de energía y nitrógeno fermentables, adecuados en cantidad y aprovechables de modo sincronizado para garantizar así la disponibilidad oportuna de nitrógeno e hidratos de carbono para el crecimiento microbiano efciciente, y el flujo máximo de proteína metabolizable al tracto posterior (1-3).

Diversas fuentes de nitrógeno no proteico (NNP) en forma elemental han sido incorporadas en programas de suplemento nutricional de vacunos en pastoreo; sin embargo, la cantidad empleada es limitada debido a que la hidrólisis puede ocurrir a una tasa superior a la utilización del amonio por los microorganismos ruminales, lo que resulta en un aumento en la absorción del amoníaco ruminal y en la posterior conversión a urea en el tejido hepático $(4,5)$. Este proceso demanda un gasto calórico para los rumiantes de 0.2 Mcal de energía neta de lactación por cada $16 \mathrm{~g}$ de exceso de nitrógeno (N) ingerido (1). Adicionalmente, un incremento del $\mathrm{N}$ ureico en plasma puede asociarse con el deterioro en el comportamiento reproductivo del animal (3), y un impacto negativo al medio ambiente debido a la excesiva excreción urinaria de N (5).

Para controlar la disponibilidad de nitrógeno en el ambiente ruminal, y facilitar el ajuste entre su disponibilidad y la energía dietaria, se ha propuesto el uso de fuentes no proteicas de liberación controlada de nitrógeno ( $\mathrm{NnpLC}$ ); entre las que se encuentran el isobutiraldehido monourea, la acetilurea, el biuret, la urea protegida con aceite de linaza y la urea tratada con formaldehido, entre otras $(4,6,7)$.

Recientemente, se ha utilizado el recubrimiento o encapsulado de partículas de urea con una película de polímeros de degradación lenta que reducen la solubilidad del material; generando una tasa menor de liberación de nitrógeno $(8,9)$. Así cuando se suplementa la ración con NnpLC, disminuyen las concentraciones iniciales de nitrógeno amoniacal $\left(\mathrm{N}-\mathrm{NH}_{3}\right)$ en el medio ruminal y se regula su ingreso al flujo sanguíneo; lo que acorta el tiempo durante el cual el rumen evidencia exceso ó déficit de nitrógeno. Lo anterior deriva en una mayor síntesis de proteína microbiana y consecuentemente, en un incremento en la digestión de la pared celular y producción de ácidos grasos volátiles (AGV), promoviendo mayor eficiencia en el funcionamiento del rumen (9).

El objetivo de esta investigación fue evaluar el efecto de la incorporación de una fuente no protéica de liberación controlada de nitrógeno en la dieta de vacas de doble propósito, sobre parámetros de la fermentación ruminal $(\mathrm{pH}$, concentración de $\mathrm{N}-\mathrm{NH}_{3}$ y producción de AGV) y la degradabilidad ruminal aparente in situ de un heno de Cynodon dactylon. 
Ojeda - Efecto liberación controlada de nitrógeno sobre la fermentación y degradabilidad 3135

\section{MATERIALES Y MÉTODOS}

Ubicación. El estudio se realizó en la Facultad de Agronomía de la Universidad Central de Venezuela (10016'24.6" $\mathrm{N}$ y 67036'30.2" O), localizada en Maracay, Aragua. Las instalaciones se ubican en un área de bosque seco tropical, a una altura de $461 \mathrm{msnm}$ y registros medios de $25.3^{\circ} \mathrm{C}$ de temperatura, $75.1 \%$ de humedad relativa y $1035.2 \mathrm{~mm}$ de precipitación anual (10).

Animales y manejo. Se emplearon 4 vacas con cánula ruminal permanente y peso vivo de 422 $\pm 93.5 \mathrm{~kg}$, confinadas en corrales individuales semitechados de $16 \mathrm{~m}^{2}$ con comederos y bebederos individuales, estos últimos con suministro de agua ad libitum. Los animales fueron alimentados con una ración base de heno de Cynodon dactylon, y suplementados una vez al día (06:00 h) con $1 \mathrm{~kg}$ de melaza de caña de azúcar y $55 \mathrm{~g}$ de una mezcla mineral comercial. Se utilizó un diseño en Cuadrado Latino 4x4, cada uno con períodos de 17 días (14 de adaptación a las raciones experimentales y 3 de evaluación), para evaluar 4 tratamientos experimentales, a saber:

Control: animales con ración base.

Urea: animales con ración base y adición de $150 \mathrm{~g} /$ día de urea.

Urea/NnpLC: animales con ración base y sustitución del $50 \%$ del aporte de nitrógeno vía urea por NnpLC (75 g urea y $91.7 \mathrm{~g}$ NnpLC).

NnpLC: animales con ración base y adición de 183 g /día de NnpLC.

La adición de NNP se efectuó a razón de 69.9 g N/día, partiendo de urea perlada comercial (466 g N/kg) y una fuente de NnpLC (381 g N/ $\mathrm{kg}$ ) suministrada por Alltech Venezuela S.C.S. (Valencia, Venezuela), la cual consistió de urea recubierta con un polímero poroso biodegradable que genera un patrón de liberación controlada del nitrógeno.

Muestreo ruminal. El día 15 de cada período experimental, y a partir del filtrado con liencillo de una fracción del contenido del rumen, se colectó una muestra del líquido ruminal inmediatamente antes del suministro de suplemento, y a las $1,3,6,9,12$ y 18 h luego de ofrecer el mismo. Una vez registrado el $\mathrm{pH}$, se tomaron $30 \mathrm{ml}$ de dicha muestra y se les adicionó ácido sulfúrico al $97 \%$ hasta alcanzar un $\mathrm{pH}$ inferior a 4 , almacenando a $-4^{\circ} \mathrm{C}$ este material para el posterior análisis de $\mathrm{N}-\mathrm{NH}_{3}$ en la fase de destilación del método de Kjeldahl (11), y determinación de la concentración molar de los ácidos acético, propiónico y butírico, empleando un cromatógrafo de gases (PYE UNICAM PU-4500) con ácido isocaproico como estándar interno (12).

Degradabilidad in situ y consumo. Siguiendo la técnica de la bolsa de nylon (13), $5 \mathrm{~g}$ de heno molido en una criba de $2.5 \mathrm{~mm}$ fueron introducidos en bolsas de nylon $(7 \times 14 \mathrm{~cm}$ y poro de $50 \mu \mathrm{m}$ ) e incubadas en el rumen por duplicado para cada tiempo y animal. Dichas bolsas se retiraron del rumen a las $0,3,6$, $12,24,48,72$ y $96 \mathrm{~h}$ y fueron lavadas con agua, para posteriormente ser deshidratadas durante 48 h en estufa con circulación de aire a $100^{\circ} \mathrm{C}$. Las muestras fueron evaluadas (14) de acuerdo al análisis químico proximal, calcio y fósforo; mientras que la fibra en detergentes neutro (FND) y ácido (FAD) se determinó de acuerdo a lo descrito por Van Soest et al (15). La degradabilidad in situ de la materia orgánica y FND se obtuvo por diferencia entre el peso de la muestra introducida en la bolsa y el remanente en cada tiempo de incubación. La fracción soluble de la materia seca contenida en las bolsas fue obtenida luego de mantenerlas en agua a $38^{\circ} \mathrm{C}$ durante $1 \mathrm{~h}$.

El tiempo al cual la mitad de la fracción de materia seca potencialmente degradable desapareció de la bolsa $\left(T_{1 / 2}\right)$ fue calculado mediante la ecuación $T_{1 / 2}(h)=\ln 2 / c$, donde " $c$ " representó la tasa de degradación derivada de la pendiente obtenida por la representación gráfica de la desaparición del material (16).

El consumo diario de heno y del suplemento, estimados a partir de la diferencia de peso entre lo ofertado y rechazado, se obtuvieron del día 7 al 14 de cada período. La relación proteína:energía (P:E) fue calculada de acuerdo a lo propuesto por Castillo et al (17), considerando los gramos de nitrógeno degradable generados a partir de la fermentación de un kilogramo de materia orgánica ( $\mathrm{g} \mathrm{N}$ ferm/kg MO deg), tomando para el cálculo los valores de degradabilidad in vitro.

Análisis estadístico. La información fue examinada empleando el software SAS (18). El consumo de materia seca y su degradabilidad in situ se analizaron por medio de ANOVA para Cuadrado Latino 4x4 (PROC MIXED), de acuerdo al modelo lineal aditivo que se detalla a continuación:

$Y_{i j k}=\mu+A_{i}+P_{j}+T_{k}+\epsilon_{i j k}$

En donde $\mu$ es la media general; $\mathrm{A}, \mathrm{P}$ y $\mathrm{T}$ los efectos debido al animal, período y tratamiento, respectivamente; $y \in$ el término de error (distribuido normalmente con media $=0$ y 
varianza constante). El $\mathrm{pH}$ ruminal y la concentración de $\mathrm{N}-\mathrm{NH}_{3}$ fueron analizados como un Cuadrado Latino con medidas repetidas, considerando el animal como efecto aleatorio. Las diferencias significativas $(p<0.05)$ entre medias fueron evaluadas a través de la prueba de comparaciones múltiples de Tukey.

\section{RESULTADOS}

Composición química. El heno de Cynodon dactylon (Tabla 1 ) presentó un aporte de nitrógeno limitado $(0.77 \% \mathrm{~N})$ y un contenido elevado de pared celular (78.4\% FND), similar al tipo de recursos fibrosos frecuentemente empleados para animales en pastoreo durante la época seca en sabanas tropicales (11). La mezcla mineral exhibió un balance de sus fracciones de acuerdo a las necesidades sugeridas para el tipo de animal en evaluación (3).

Tabla 1. Composición química de los alimentos.

\begin{tabular}{lccc}
\hline \multicolumn{1}{c}{ Fracciones } & Heno & $\begin{array}{c}\text { Composición química (\% BS) } \\
\text { Melaza } \\
\text { de caña }\end{array}$ & $\begin{array}{c}\text { Mezcla } \\
\text { Mineral }^{1}\end{array}$ \\
\hline Materia seca & 87.5 & 73.7 & 96.0 \\
Proteína cruda & 4.8 & 4.3 & \\
Extracto etéreo & 1.8 & 0.12 & \\
FND & 78.4 & & \\
FAD & 45.0 & & \\
Cenizas & 5.8 & 9.8 & 15.8 \\
Calcio & 0.71 & 0.74 & 8.2 \\
Fósforo & 0.33 & 0.07 & \\
\hline
\end{tabular}

${ }^{1}$ Composición indicada por el fabricante: $9 \% \mathrm{Na}, 0.5 \% \mathrm{Mg}, 0.6 \% \mathrm{~S}, 0.5 \%$ $\mathrm{Zn}, 0.25 \% \mathrm{Cu}, 20 \mathrm{ppm} \mathrm{Se}, 80 \mathrm{ppm} \mathrm{I}$; entre otros.

Consumo. El consumo de materia seca (Tabla 2) no fue afectado por los tratamientos ( $p>0.05$ ); alcanzando $8.2 \pm 0.31 \mathrm{~kg} \mathrm{MS} /$ animal/ día, equivalentes al $1.9 \%$ del peso vivo de los animales.

Tabla 2. Consumo y parámetros de la fermentación ruminal de vacunos suplementados con urea y/o una fuente no proteica de liberación controlada de nitrógeno.

\begin{tabular}{|c|c|c|c|c|}
\hline \multirow[b]{2}{*}{ Variables } & \multicolumn{4}{|c|}{ Tratamientos } \\
\hline & Control & Urea & $\begin{array}{l}\text { Urea/ } \\
\text { NnpLC }\end{array}$ & NnpLC \\
\hline Consumo (kg/día) & 8.0 & 8.6 & 8.2 & 7.9 \\
\hline $\mathrm{pH}$ & 6.3 & 6.3 & 5.9 & 6.2 \\
\hline $\mathrm{N}$ amoniacal (mg/L) & $19.1^{c}$ & $166.7^{\mathrm{b}}$ & $181.6^{b}$ & $281.8^{a}$ \\
\hline \multicolumn{5}{|l|}{ AGV (mmol/L) } \\
\hline Acetato & $4.8^{\mathrm{b}}$ & $6.0^{a}$ & $6.2^{\mathrm{a}}$ & $6.1^{\mathrm{a}}$ \\
\hline Propionato & $1.0^{b}$ & $1.2^{\mathrm{b}}$ & $1.4^{\mathrm{a}}$ & $1.4^{\mathrm{a}}$ \\
\hline Butirato & 0.4 & 0.4 & 0.4 & 0.5 \\
\hline \multicolumn{5}{|l|}{ AGV (\%) } \\
\hline Acetato & $77.4^{b}$ & $78.9^{a}$ & $77.5^{b}$ & $76.3^{b}$ \\
\hline Propionato & $16.2^{b}$ & $15.8^{b}$ & $17.5^{\mathrm{a}}$ & $17.5^{\mathrm{a}}$ \\
\hline Butirato & 6.4 & 5.3 & 5.0 & 6.3 \\
\hline
\end{tabular}

Promedios con letras diferentes entre columnas, son estadísticamente diferentes $(p<0.05)$
Parámetros de la fermentación ruminal. La incorporación de fuentes suplementarias de NNP no modificó $(p>0.05)$ el $p H$ del líquido ruminal, el cual se ubicó entre 5.9 y 6.3 , con un valor medio de $6.2 \pm 0.19$.

Comparado con el control (19.1 mg N-NH$/ 2$ ), se observó un aumento $(p<0.01)$ del contenido de $\mathrm{N}-\mathrm{NH}_{3}$ en el líquido ruminal de 9.1 veces para los tratamientos Urea y Urea/NnpLC, y de 14.8 veces en el tratamiento NnpLC. Los tratamientos que incluyeron suplementación con NNP generaron un incremento sostenido del $\mathrm{N}-\mathrm{NH}_{3}$ durante las primeras 3 a $6 \mathrm{~h}$ posteriores a la ingesta del suplemento (Figura 1), alcanzando una media de $375.9 \mathrm{mg} \mathrm{N}-\mathrm{NH}_{3} / \mathrm{L}$. Sin embargo, la suplementación con NnpLC garantizó mayor uniformidad en la concentración ruminal de $\mathrm{N}-\mathrm{NH}_{3}$ a lo largo del período en evaluación.

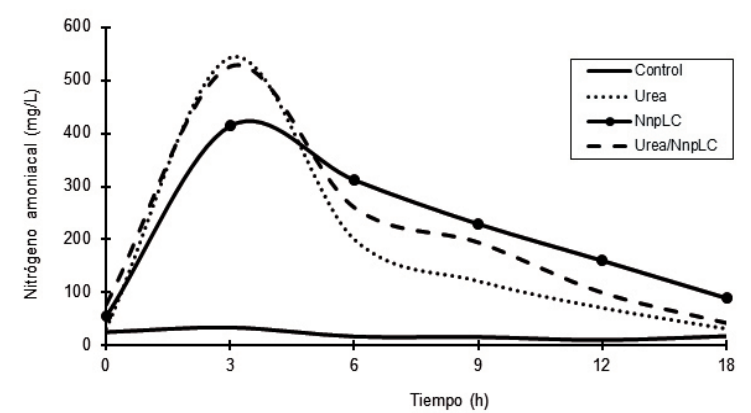

Figura 1. Comportamiento del nitrógeno amoniacal ruminal en vacunos suplementados con urea y una fuente no proteica de liberación controlada de nitrógeno (NnpLC).

En general, todos los tratamientos presentaron un perfil de fermentación ruminal de carácter acetogénico $(77.6 \pm 1.3 \%)$, con una mayor $(p<0.05)$ concentración de acetato en los tratamientos que incluyeron suplementación con fuentes de NNP $(6.1 \pm 0.1 \mathrm{mmol} / \mathrm{L})$ respecto al control $(4.8 \mathrm{mmol} / \mathrm{L})$. No hubo diferencias en la concentración de butirato $(0.4 \pm 0.1 \mathrm{mmol} / \mathrm{L})$, aunque respecto a los tratamientos restantes, la inclusión de NnpLC generó un incremento $(p<0.05)$ en la fracción de ácido propiónico tanto en términos absolutos (27.3\%), como en su proporción molar (9.4\%).

Degradabilidad in situ. La degradabilidad aparente de la materia orgánica y la FND no se vieron afectadas por los tratamientos evaluados $(p>0.05)$. Sin embargo, el $T_{1 / 2}$ se redujo $(p<0.05)$ un $13.2 \%$ en el tratamiento NnpLC (Tabla 3). Comparado con el control $(7.67 \mathrm{~g} \mathrm{~N}$ ferm $/ \mathrm{kg} \mathrm{MO}$ deg), la incorporación a la ración de las fuentes de NNP evaluadas mejoró $(\mathrm{p}<0.01)$ la relación $\mathrm{P}: \mathrm{E}$ (22.0 $\pm 0.76 \mathrm{~g} \mathrm{~N}$ ferm $/ \mathrm{kg} \mathrm{MO} \mathrm{deg).}$ 
Tabla 3. Degradabilidad in situ de Cynodon dactylon y relación proteína:energía ( $\mathrm{g} \mathrm{N}$ ferm/ $\mathrm{kg} \mathrm{MO}$ deg) en vacunos suplementados con urea y/o una fuente no proteica de liberación controlada de nitrógeno.

\begin{tabular}{|c|c|c|c|c|}
\hline \multirow[b]{2}{*}{$\begin{array}{c}\text { Variables } \\
\text { (\% BS) }\end{array}$} & \multicolumn{4}{|c|}{ Tratamientos } \\
\hline & Control & Urea & Urea/NnpLC & NnplC \\
\hline \multicolumn{5}{|c|}{ Materia orgánica } \\
\hline $48 \mathrm{~h}$ & 55.6 & 56.6 & 45.1 & 54.8 \\
\hline $72 \mathrm{~h}$ & 60.3 & 59.3 & 59.8 & 57.9 \\
\hline \multicolumn{5}{|l|}{ FND } \\
\hline $48 \mathrm{~h}$ & 33.8 & 33.0 & 27.5 & 29.7 \\
\hline $72 \mathrm{~h}$ & 39.8 & 37.7 & 33.5 & 39.6 \\
\hline$T_{1 / 2}(h)^{3}$ & $56.5^{\mathrm{a}}$ & $52.2^{\mathrm{a}}$ & $58.4^{\mathrm{a}}$ & $48.1^{b}$ \\
\hline$P: E$ & $7.67^{\mathrm{b}}$ & $21.2^{\mathrm{a}}$ & $22.7^{\mathrm{a}}$ & $22.2^{\mathrm{a}}$ \\
\hline
\end{tabular}

Promedios con letras diferentes entre columnas, son estadísticamente diferentes $(p<0.05)$

\section{DISCUSIÓN}

Consumo. El consumo de alimento fue similar entre tratamientos $(p>0.05)$, y estuvo localizado en el rango referido en la literatura para recursos fibrosos con elevada participación de fracciones refractarias en su pared celular (19). La ausencia de una mejora del consumo voluntario producto de la suplementación nitrogenada puede deberse a un marcado efecto de distención ruminal asociado al alto contenido de pared celular del recurso fibroso evaluado, o a posibles efectos tóxicos derivados del metabolismo de nitrógeno excedentario.

En este sentido, la ingesta de urea o su equivalente en NnpLC se sitúo en $18.2 \pm 0.77 \mathrm{~g} / \mathrm{kg}$ de materia seca ingerida, mientras que en términos de proteína cruda ( $\mathrm{N} * 6.25)$, la ración suministró 94.0 $\pm 2.8 \mathrm{~g} \mathrm{PC} / \mathrm{kg}$ ración. Estos valores son similares a lo establecido como límite $(20 \mathrm{~g} / \mathrm{kg}$ MS y 105 g PC/kg ración, respectivamente) para observar efectos inhibitorios en el consumo voluntario de recursos fibrosos como consecuencia de los metabolitos resultantes del catabolismo del NNP dietario $(20,21)$.

Parámetros de la fermentación ruminal. En general, los valores de $\mathrm{pH}$ ruminal son inferiores al rango deseable (6.6 a 7.0) para una mayor eficiencia en el crecimiento de los microorganismos celulolíticos (19) y se consideran resultado del aporte de carbohidratos rápidamente fermentables por la melaza de caña.

La concentración de $\mathrm{N}-\mathrm{NH}_{3}$ en el tratamiento control a lo largo del día es inferior al óptimo (100 $\mathrm{mg} \mathrm{N}-\mathrm{NH}_{3} / \mathrm{L}$ ) reportado para maximizar el consumo y la utilización de la materia orgánica en el rumen (22); mientras que los tratamientos Urea, Urea/NnpLC y NnpLC se mantienen por encima de dicho valor durante $10.5,12.3$ y 18 h; respectivamente.

En un ambiente ruminal con disponibilidad de carbohidratos fermentables, mantener niveles óptimos de $\mathrm{N}-\mathrm{NH}_{3}$ en el medio ruminal durante la mayor parte del día, genera una eficiencia elevada en el uso de la energía para crecimiento microbiano $\left(\mathrm{Y}_{\mathrm{ATP}}\right)$, y por tanto, un incremento de la proteína metabolizable. Esto ha sido demostrado por Tikofsky y Harrison (8), quienes obtuvieron un incremento de $40.1 \%$ $(p=0.07)$ en la síntesis de proteína microbiana por unidad de materia seca verdaderamente digestible al evaluar la inclusión de esta misma fuente de NnpLC en sustitución de urea en la ración (18.2 vs $13.0 \mathrm{~g} \mathrm{~N}$ bacterial/kg MSdeg; respectivamente).

La curva descrita por la concentración ruminal de $\mathrm{N}-\mathrm{NH}_{3}$ muestra una mayor estabilidad en el tiempo del contenido de amonio, y se evidencia un "espacio vertical" entre las líneas de los tratamientos Urea/NnpLC y NnpLC, respecto a la urea. Esto repercute en una menor cantidad de nitrógeno liberado al medio ruminal durante las primeras $5 \mathrm{~h}$ luego de ingerida la fuente de NnpLC, y una mayor persistencia de la concentración de amonio ruminal entre las 5 y 18 h. Este comportamiento se traduce en menores riesgos de toxicidad y menor pérdida de energía para la excreción del nitrógeno úrico generado en el metabolismo intermediario $(5,9)$; además, de una reducción de la contaminación ambiental por la emisión de compuestos nitrogenados al entorno (8).

Aunque en general el patrón de producción de AGV ruminales fue de carácter acetogénico, una mayor proporción de ácido propiónico en los tratamientos urea/NnpLC y NnpLC tuvo consecuencias positivas en la repuesta de animales a pastoreo, ya que este $A G V$ es el único involucrado en la síntesis neta de glucosa a partir de su carboxilación a metilmalonil-CoA, por lo que contribuye a suplir la demanda de este carbohidrato para los procesos catabólicos (generación de energía) y anabólicos (síntesis de aminoácidos, glucógeno, lactosa, glicerol y ácidos grasos) en el rumiante (23).

Degradabilidad in situ. La ausencia de efecto sobre la degradabilidad de la materia orgánica y la FND coincide con estudios previos (8), y con la respuesta observada en el consumo voluntario. La reducción en el $T_{1 / 2}$ en el tratamiento NnpLC es debido a una mejor distribución en el tiempo del nitrógeno disponible al medio ambiente ruminal $(8,9)$. Independientemente del tratamiento, la inclusión de NnpLC incrementó 
la relación $P$ : E en rumen, aunque éstas fueron ligeramente inferiores al rango de $25-30 \mathrm{~g} \mathrm{~N}$ ferm/kg MO deg establecido como óptimo para un funcionamiento ruminal eficiente (17).

La incorporación de una fuente de NnpLC a la dieta de vacas de doble propósito alimentadas con heno (Cynodon dactylon), sola o en combinación con urea, no alteró el consumo voluntario, el pH ruminal, la degradabilidad in situ de la materia orgánica y el FND; sin embargo, se observó una mayor estabilidad en el tiempo de la concentración de $\mathrm{N}-\mathrm{NH}_{3}$ se modificó el perfil de AGV hacia un patrón gluconeogénico, se redujo el $\mathrm{T}_{1 / 2}$ de degradación de la materia seca y se generó un mejor ajuste de la relación $\mathrm{P}$ : E. Se requiere desarrollar estudios de respuesta animal en vacunos a pastoreo de recursos fibrosos en el medio tropical.

\section{Agradecimientos}

A Alltech Venezuela S.C.S., por la financiación parcial de la presente investigación.

\section{REFERENCIAS}

1. Kaswari T, Lebzien P, Flachowsky G, Meulen $U$. Studies on the relationship between the synchronization index and the microbial protein synthesis in the rumen of dairy cows. Anim Feed Sci Technol 2007; 139:1-22.

2. Dewhurst RJ, Davies DR, Merry RJ. Microbial protein supply from the rumen. Anim Feed Sci Technol 2000; 85:1-21.

3. National Research Council (NRC). The nutrient requirement of dairy cattle. Washington (DC): National Academy Press; 2001.

4. Hristov AN, Ropp JK, Grandeen $\mathrm{KL}$, Abedi S, Etter RP, Melgar A et al. Effect of carbohydrate source on ammonia utilization in lactating dairy cows. J Anim Sci 2005; 83:408-421.

5. Zanton GI, Heinrichs AJ. Analysis of nitrogen utilization and excretion in growing dairy cattle. J Dairy Sci 2008; 91:1519-1533.

6. Mathison GW, Soofisiawash R, Worsley $M$. The potential of isobutyraldehydemonourea (propanal, 2-methyl-monourea) as a non-protein nitrogen source for ruminant animals. Can J Anim Sci 1994; 74:665-674.

7. Löest CA, Titgemeyer EC, Drouillard JS, Lambert BD, Trater AM. Urea and biuret as non-protein nitrogen sources in cooked molasses blocks for steers fed prairie hay. Anim Feed Sci Technol 2001; 94:115-126.
8. Tikofsky J, Harrison GA. Optigen $®$ II: improving the efficiency of nitrogen utilization in the dairy cow. En: Lyons TP., editor. Proceedings of the Alltech's 22nd Annual Symposium; 2007 Apr 2226; Lexington, Kentucky. Nottingham University Press, UK. 2007. p. 373-382

9. Galo E, Emanuele SM, Sniffen CJ, White JH, Knapp JR. Effects of a polymer-coated urea product on nitrogen metabolism in lactating Holstein dairy cattle. J Dairy Sci 2003; 86:2154-2162.

10. INIA. Informe de Estación Climatológica. Unidad Agroclimatológica, Venezuela: Instituto Nacional de Investigaciones Agrícolas; 2010: 13-15.

11. Preston TR. Tropical animal feeding. Animal Production and Health Paper No 126. Roma: FAO; 1995.

12. Tajima K, Nonaka I, Higuchi K, Takusari $\mathrm{N}$, Kurihara M, Takenaka A. Influence of high temperature and humidity on rumen bacterial diversity in Holstein heifers. Anaer 2007; 13:57-64.

13. Ørskov EF, Hovwell D, Mould F. The use of the nylon bag technique for the evaluation of feedstuff. Trop Anim Prod 1980; 5:195-213.

14. Association of Official Analytical Chemist (AOAC). Official Methods of Analysis. 18 ed. Arlington (VA): AOAC Intl; 2007. 
Ojeda - Efecto liberación controlada de nitrógeno sobre la fermentación y degradabilidad 3139

15. Van Soest PJ, Robertson JB, Lewis BA. Methods for dietary fiber, neutral detergent fiber and non-starch polysaccharides in relation to animal nutrition. J Dairy Sci 1991; 74: 3583-3597.

16. Kempton TJ. El uso de las bolsas de nylon parta caracterizar el potencial de degradabilidad de los alimentos para el rumiante. Produc Anim Trop 1980; 5:115-126.

17. Castillo AR, Kebreab E, Beever DE, Barbi $J H$, Sutton JD, Kirby HC, France J. The effect of protein supplementation on nitrogen utilization in lactating dairy cows fed grass silage diets. J Anim Sci 2001; 79:247-253.

18. SAS. User's Guide: Statistics, Version 9.1 Cary (NC): SAS Inst. Inc.; 2002.

19. Van Soest P. Nutritional Ecology of the Ruminant. 2 ed. Ithaca, (NY): Cornell University Press; 1994.
20. Tedeschi LO, Baker MJ, Ketchen DJ, Fox DG. Performance of growing and finishing cattle supplemented with a slow-release urea product and urea. Can J Anim Sci 2002; 82:567-573.

21. Rezende LHGS, Albertini TZ, Detmann E, Tomich TR, Franco GL, Lempp B et al. Intake and digestibility of palisade grass hay by beef cattle supplemented with a mixture containing ammonium sulphate, casein and urea. R Bras Zootec 2008; 37:717-723.

22. Chandrasekharaiah $M$, Thulasi $A$, Suresh KP, Sampath KT. Rumen degradable nitrogen requirements for optimum microbial protein synthesis and nutrient utilization in sheep fed on finger millet straw (Eleucine coracana) based diet. Anim Feed Sci Technol 2011; 163: 130-135.

23. Udén $P$, Danfaer $A$. Modeling glucose metabolism in the dairy cow. A comparison of two dynamic models. Anim Feed Sci Technol 2008; 143:59-69. 